\title{
Environmentally safe techniques in the recycling of mercury-containing materials
}

\author{
I. Maslennikova ${ }^{1}, \mathrm{~V} . \mathrm{Kovalev}^{2}$, O. Eronko ${ }^{1} \&$ T. Grishchenko ${ }^{1}$ \\ ${ }^{1}$ Department of Environmental Management, \\ Saint-Petersburg State University of Economics, Russia \\ ${ }^{2}$ Laboratory of Hydrometallurgy, CJSC "Polymetal Engineering", \\ Saint-Petersburg, Russia
}

\begin{abstract}
Protection of the environment can be achieved by creating conditions that ensure the complete absence, or at least minimal content, of hazardous substances in industrial waste. Therefore, in production processes where the main and/or additive product is material with mercury content, the prevention of mercury vapor emissions into the atmosphere is an essential prerequisite for safety and environmental protection. However, achieving these indispensable safety conditions requires significant energy input.

Detailed analysis of existing techniques and equipment for mercury extraction from mercury-containing and mercury-contaminated materials as well as plastics containing waste showed that all of the proposed methods are energy-intensive and cannot entirely remove mercury vapor from the emissions.

A new technique of the thermal demercurization of mercury-containing materials has been offered. The original feature of the proposed technique is the distillation and condensation of mercury vapor within an inert gas flow circulating in the closed loop system. The offered technique ensures a substantial reduction in energy consumption while cooling the mercury vapor for its condensation and almost entirely eliminates mercury emissions into the atmosphere.

Keywords: recycling of mercury-containing materials, environmental protection, energy consumption, thermal demercurization, inert gas flow, closed gas loop system, closed liquid loop system.
\end{abstract}




\section{Introduction}

The prevention of mercury vapour emissions into the atmosphere is an essential prerequisite for personal safety and environmental protection during production processes where the main or additive product is a material with mercury content.

During the processing of mercury-containing materials it is often necessary to begin by extracting the mercury from the material [1] and this process may result in environmental pollution.

The existing techniques and equipment for mercury extraction from mercurycontaining and mercury-contaminated materials as well as plastics containing waste are described in [1-3]. However, when using the methods proposed in the specified references, a significant drawback is the significant level of mercury in the emissions.

By creating conditions that ensure the complete absence of hazardous substances, or at least their minimal content, the environment can be protected. Therefore, the challenge is to reduce emissions of mercury vapour into the atmosphere.

In the technique proposed by the authors earlier [4], during the heat demercurization of mercury-containing materials, including the stages of heating the material and the distillation of mercury and condensation of its vapour in a refrigerating trap, the problem is resolved by carrying out the distillation and condensation of mercury vapour within an inert gas flow circulating within a closed loop system.

\section{Experimental}

The proposed apparatus for thermal demercurization of mercury-containing materials consists of a container with the material being processed being linked to valved pipelines with a heater, a trap and a pump. The apparatus is equipped with a jet pump, situated in front of the trap, designed in the form of a separator with a spray trap and a heat exchanger connected to the jet pump and the separator via a pump. All the elements of the apparatus form two closed-loop systems: the closed-loop gas flow system, which comprises a jet pump, a separator, a heater and a container with work material, and the liquid phase closed-loop system consisting of a separator, a pump, a heat exchanger and a jet pump.

The scheme for the demercurization of zinc precipitate is shown in Figure 1.

In the method, the process of distillation is implemented in an inert gas flow. An inert gas which does not react chemically with mercury under the conditions of thermal demercurization is used.

The mercury evaporates in a flow of hot inert gas. In the proposed apparatus the inert gas circulates in a closed-loop gas flow system, while the liquid circulates in the liquid phase closed-loop system.

The apparatus comprises a vertical cylindrical container with the processed material - the retort 1 equipped with a hermetic cover. Inside the retort case there 


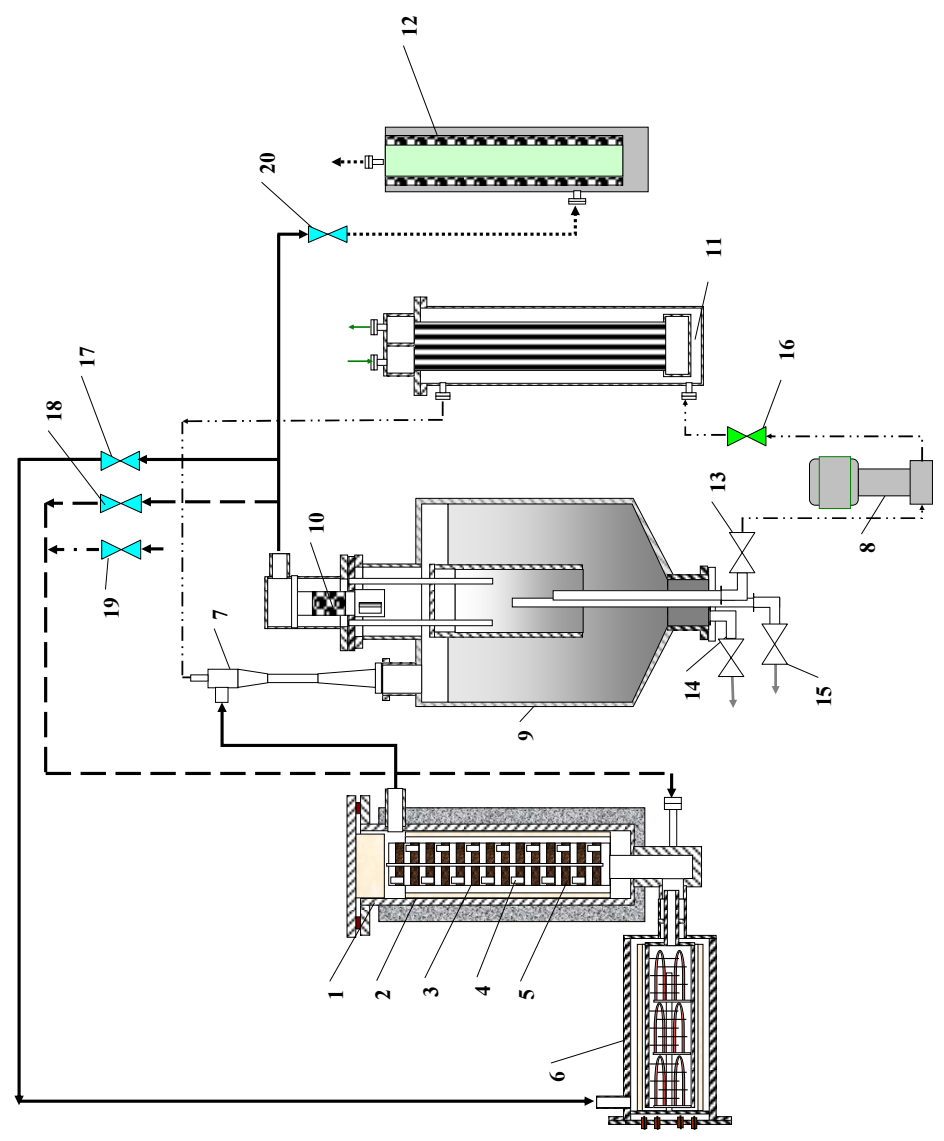


is a thermos 2, where the cassette 3 with plates 4 filled with zinc precipitate 5 is installed. The apparatus also includes an electrical heater 6, a jet pump 7 to ensure gas circulating, a centrifugal pump 8, the trap for mercury extraction designed in the form of a separator 9 with a spray trap 10 disposed in its upper part, a heat exchanger 11 connected to the jet pump 7 and the separator via a pump 8. At the outlet there is an adsorber 12 to absorb mercury vapour from the gaseous phase, which is used only when blowing the apparatus' gas volume by air before unloading the cassette. In the bottom section of the separator 9 there are output ducts with attached fittings: output duct 13 is for circulation during the liquid phase, output duct 14 is for the periodic discharge of mercury, 15 is for periodic water drainage. All the elements of the apparatus are linked by the pipelines for the gas and liquid phase transfer equipped with valves 16, 17, 18, 19 and 20.

\section{Results and discussion}

The operation of the equipment in accordance with proposed method is carried out as follows: zinc precipitate is loaded in the plates 4 , which are then packed into the cassette 3 and installed in the retort 1 . Then the centrifugal pump 8, which delivers the liquid phase (water) into the heat exchanger 11 and then into the jet pump 7, is turned on. The jet pump ensures the circulation of the inert gas through the equipment. During the process of mercury distillation valve 17 is opened and valves 18, 19 and 20 are closed. The flow of inert gas (air) heated to $600-700{ }^{\circ} \mathrm{C}$ is supplied from the heater 6 into the retort 1 , where it coats all the layers of the plates with zinc precipitate. The gas containing mercury vapour is ejected by the jet pump 7 from the retort 1 to the separator 9 and is accompanied by an increase in the gas phase pressure. Within the jet pump 7 an intense interaction between the gas-vapour and liquid phases occurs. As a result of cooling, the mercury vapours condense and the separation of gaseous and liquid phases then takes place in separator 7. The liquid phase from the separator 9 via the outlet 13 is supplied by the centrifugal pump 8 firstly to the heat exchanger 11 and then into the jet pump 7. In the heat exchanger, the liquid phase moves into the shell side space and is cooled with tap water flowing into the tube space. The inert gas flows into the spray trap 10, where it is separated from the condensed moisture, then passes through the electric heater 6 and flows back into the retort 1 .

During the cooling phase of retort 1 the circulation of inert gas is carried throughout a shortened loop: jet pump 7 - separator 9 - valve 17 - heater 18 retort 1 - jet pump 7. Valves 17, 18 and 19 are closed. When using the shortened circuit an inert gas from the separator 9 does not pass through the electric heater 6 , which allows it to retain a sufficiently high temperature while the retort is cooled. In this case cooling of the electric heater takes place only via heat loss through the thermal insulation.

Mercury accumulates in the bottom of separator 9 and periodically discharges through outlet 14 and periodically water drains out through outlet 15 . Separation of splatters from the gas phase is carried by the spray trap 10 and captured fluid 
is returned to the bottom of the separator 9. The inert gas flow is controlled by valve 16 by changing the flow rate and pressure of the liquid phase at the inlet of the jet pump 7.

After completion of the mercury distillation process the heater 6 is turned off and the precipitate in the retort is cooled by an inert gas circulating in the shortened loop. Before unloading the cassette 3 out the retort, the gas volume is blown by air through the adsorber 12, whereby the gases emitted into the atmosphere are additionally purified of mercury vapour and other harmful substances. Air is fed into the apparatus through valve 19. The waste gases are directed into the adsorber through valve 20. In blowing mode valves 17 and 18 are closed.

There are no emissions during this process because the inert gas and the liquid phase are circulating in the hermetic closed-loop systems. An air streamlet emitted into the atmosphere during the purging of apparatus initially lets through the carbon adsorber 12, where it is purified of mercury vapour and other harmful substances.

In contrast to known methods [5, 6], in the proposed apparatus the mass transport of mercury (evaporation and condensation) is carried out in a gas flow circulating in a closed loop system. In order to achieve low concentrations of mercury in the emissions it is not necessary to cool the gas flow saturated with mercury vapour to a low temperature. The implementation of the condensation of mercury vapour in the jet pump 7 in the temperature range $50-75^{\circ} \mathrm{C}$ means that not only is there a reduction in the consumption of refrigerant in the heat exchanger 11 and the consumption of electricity in the heater 6 , but there is also a reduction in the required dimensions of these devices. Therefore, the proposed method virtually eliminates mercury emissions into the atmosphere while expending significantly less energy.

Reported advantages of the technique in the recycling of mercury-containing materials have been confirmed in practice when using our equipment by Bashkir gold mining company. Fig. 2 is the partially assembled apparatus for the mercury retort distillation of zinc precipitate before it is placed in the production apparatus' frame (in the background).

\section{Conclusions}

A new technique of the thermal demercurization of mercury-containing materials has been offered. The original feature of the proposed technique is the distillation and condensation of mercury vapor within an inert gas flow circulating in the closed loop system. The offered technique ensures a substantial reduction in energy consumption while cooling the mercury vapor for its condensation and almost entirely eliminates mercury emissions into the atmosphere. The advantages of the new technique in the recycling of mercury-containing materials outlined in this work have been confirmed in its practical application. 


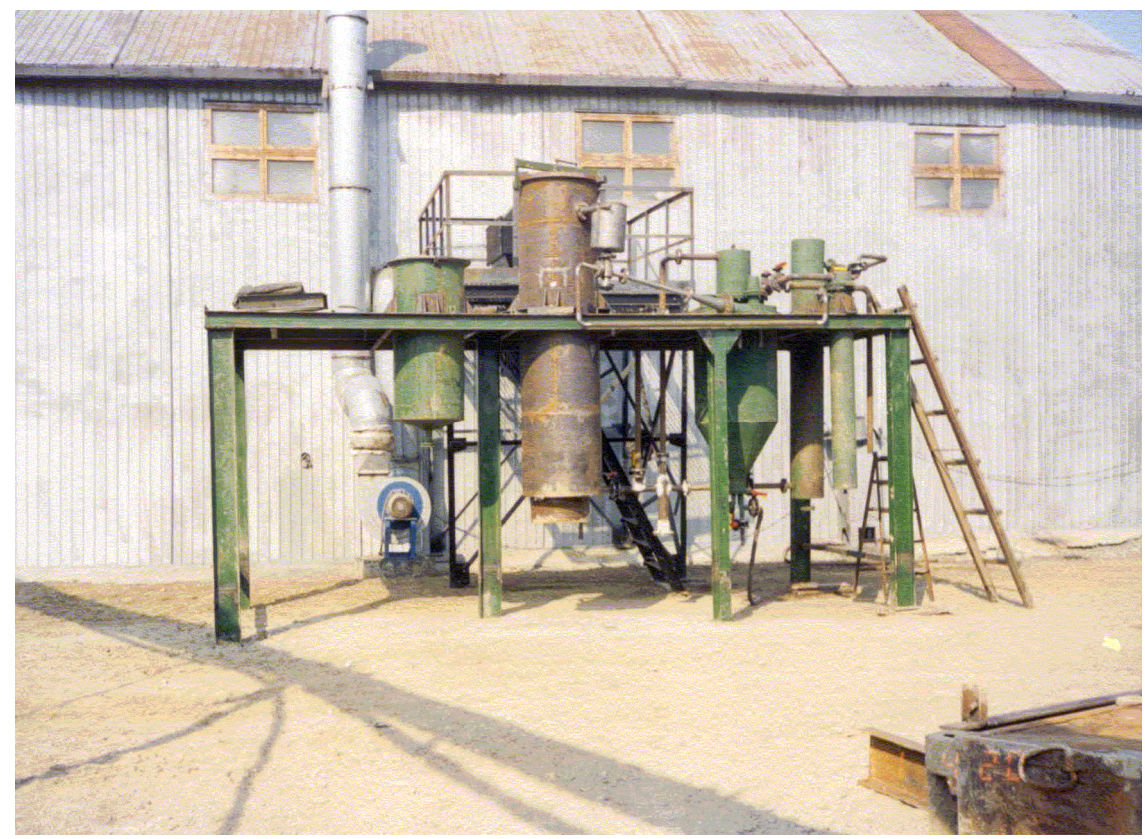

Figure 2: The industrial apparatus as used in the demercurization of zinc precipitate.

\section{References}

[1] Bat'kaev I.I., Anarbaev A.A., Tleukulov O.M. "Kiln installation for processing of mercury-containing materials", Certificate of Authorship for Innovation 1708902, January 30, 1992

[2] Jonsson G., Bjerkman E., Sikander E., "Apparatus for demercurization of wastes" Patent SU 1466665I C1, May 19, 1981

[3] Alpert V.A., Pikin A.I. "Method for thermal demercurization of material contaminated with mercury and device for its realization" 1993-08-30. Patent RU 1838440 C1, August 30, 1993

[4] Kovalev V.N., Gorbenko A.N. "Method for heat demercurization of mercury containing materials and apparatus for performing the same", Patent RU 2171304 C1, July 27, 2001

[5] Podgorbunskikh A.M, Chelin F.V. "Method of firing of mercury-containing ore" Certificate of Authorship for Innovation 1514816, October 15, 1989

[6] Djachenko Ju.G., Zakharov A.A. "Thermal chamber for treatment of solid mercury-containing wastes", Patent RU 2203971, May 10, 2003 